\title{
Human Adipose Stem Cells Cell Sheet Constructs Impact Epidermal Morphogenesis in Full-Thickness Excisional Wounds
}

\author{
M. T. Cerqueira, ${ }^{\dagger \dagger}$ R. P. Pirraco, ${ }^{\dagger \dagger}$ T. C. Santos, ${ }^{\dagger \neq}$ D. B. Rodrigues, ${ }^{\dagger \neq}$ A. M. Frias, ${ }^{\dagger \neq}$ A. R. Martins, ${ }^{\dagger, \ddagger}$ \\ R. L. Reis, ${ }^{\dagger, \ddagger}$ and A. P. Marques*, ${ }^{\dagger}, \neq$ \\ $\dagger$ 3B’s Research Group - Biomaterials, Biodegradables and Biomimetics, University of Minho, Headquarters of the European Institute \\ of Excellence on Tissue Engineering and Regenerative Medicine, AvePark4806-909, Taipas, Guimarães, Portugal \\ \# ICVS/3B’s - PT Government Associate Laboratory, Braga/Guimarães, Portugal
}

\section{Supporting Information}

ABSTRACT: Among the wide range of strategies to target skin repair/regeneration, tissue engineering (TE) with stem cells at the forefront, remains as the most promising route. Cell sheet (CS) engineering is herein proposed, taking advantage of particular cell-cell and cell-extracellular matrix (ECM) interactions and subsequent cellular milieu, to create 3D TE constructs to promote full-thickness skin wound regeneration. Human adipose derived stem cells (hASCs) CS were obtained within five days using both thermoresponsive and standard cell culture surfaces. hASCs-based constructs were then built by superimposing three CS and transplanted into full-thickness

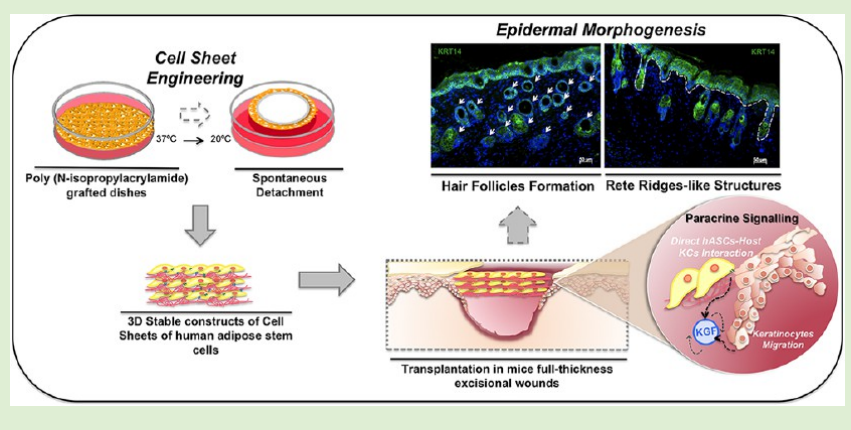
excisional mice skin wounds with delayed healing. Constructs obtained using thermoresponsive surfaces were more stable than the ones from standard cell culture surfaces due to the natural adhesive character of the respective CS. Both CS-generating strategies lead to prolonged hASCs engraftment, although no transdifferentiation phenomena were observed. Moreover, our findings suggest that the transplanted hASCs might be promoting neotissue vascularization and extensively influencing epidermal morphogenesis, mainly through paracrine actions with the resident cells. The thicker epidermis, with a higher degree of maturation characterized by the presence of rete ridges-like structures, as well as a significant number of hair follicles observed after transplantation of the constructs combining the CS obtained from the thermoresponsive surfaces, reinforced the assumptions of the influence of the transplanted hASCs and the importance of the higher stability of these constructs promoted by cohesive cell-cell and cell-ECM interactions. Overall, this study confirmed the potential of hASCs CS-based constructs to treat full-thickness excisional skin wounds and that their fabrication conditions impact different aspects of skin regeneration, such as neovascularisation, but mainly epidermal morphogenesis.

\section{INTRODUCTION}

To achieve complete healing of a cutaneous wound, a wellorchestrated sequence of mechanisms, including the complex biological and molecular events of cell migration and proliferation, extracellular matrix (ECM) deposition, and remodeling, as well as angiogenesis, is required. ${ }^{1}$ In postnatal human skin, upon cases of minor skin wounds, efficient healing occurs without treatment, whereas in cases of extensive, full thickness wounds, a rapid and effective intervention is needed to heal in a timely manner and without extensive scarring. ${ }^{2}$

Stem cells therapy has emerged as a fascinating approach for enhancing wound healing, mainly due to those cells prolonged self-renewal capacity, their ability to proliferate and differentiate into various cell types, ${ }^{3}$ and to a significant role in promoting wound vascularization, ${ }^{4,5}$ reducing wound contraction and inducing keratinization, ${ }^{4}$ thus, improving skin healing., ${ }^{6,7}$ Adipose stem cells (hASCs), in particular, represent a very attractive cell type to use in this context. hASCs secrete a range of favorable angiogenic factors, thus, explaining their ability to induce skin neovascularization. ${ }^{8,9}$ Besides, hASCs have also shown to be immune-privileged, as they lack expression of human leukocyte antigen-DR, and also to stimulate the production of anti-inflammatory cytokines. ${ }^{10}$ hASC effects are further extended to the epidermal compartment. Several studies $^{3,5,11,12}$ have shown the contribution of transplanted hASCs over epidermal components of restored skin either through differentiation and by paracrine mechanisms, ${ }^{11,13,14}$ resulting in the formation of thicker epithelium and a more effective re-epithelialization. ${ }^{15}$ In addition, hASCs hold the obvious advantages of being isolated from the adipose tissue in relative abundance using a relatively simple isolation procedure with less donor morbidity. ${ }^{16}$

General approaches for stem cell delivery, such as the direct injection of single cell suspensions, have shown encouraging

Received: July 26, 2013

Revised: $\quad$ September 27, 2013 
results in skin wound healing; ${ }^{5}$ however, in some cases, only a few of the transplanted stem cells migrate and actively participate in skin restoration, ${ }^{17}$ presumably due to the reduced residence time. ${ }^{12}$ Moreover, issues such as optimal cell number per treatment, need for repeated applications, or appropriate ECM molecule analogues for cell delivery still remain. ${ }^{18}$ An innovative alternative to both the combination of biomaterials with living cells and the injection of single cells suspensions is cell sheet engineering. This technology has been successfully applied in the context of regenerative medicine to treat and replace various tissues. ${ }^{19-21}$ This original scaffold-free TE approach takes advantage of temperature-responsive culture surfaces $^{22}$ that allow the noninvasive harvest of in vitro cultured cells as intact sheets along with their deposited extracellular matrix (ECM). This facilitates the direct transplantation to host tissues or the in vitro creation of tissue constructs by stacking individual cell sheets (CS), as the ECM acts as natural glue. Being embedded in their own matrix, cell residence time and, consequently, engraftment are significantly increased when using this approach, thus, overcoming one of the major issues of the injection of single cell suspensions. Regarding skin replacement, in particular, CS technology also presents a set of advantages when comparing with scaffolds-based approaches, due to the still unrevealed scaffolds properties that maximize skin regeneration. Several hydrogels, such as dextran, ${ }^{23}$ pullulan-collagen, ${ }^{24,25}$ and fibrin-based ${ }^{26}$ have been explored as wound healing promoters. However, fail in providing cell adhesion sites acting mostly as a temporary cell delivery vehicle, $^{24,25,27}$ instead of working as a template for matrix regeneration. Having this in consideration and knowing that CS transplantation shows high cell survival rates, ${ }^{1428}$ we hypothesized that the cellular machinery together with the deposited ECM character would impact full-thickness skin wound regeneration. hASCs-based constructs were obtained through CS engineering in a reduced time in order to assess the applicability of the method in a clinical setting and by mechanical dissociation from the culture plate to infer about a potential effect of the organized/intact nature of CSassociated ECM. Evidences that the proposed strategies yield important outcomes relevant for skin regeneration were provided after transplantation into mice full-thickness excisional skin wounds.

\section{MATERIALS AND METHODS}

2.1. Isolation and Culture of hASCs. Human subcutaneous adipose tissue was obtained from liposuction procedures performed at Hospital da Prelada (Porto), after patient's informed consent and under a collaboration protocol approved by the ethical committees of both institutions. After digestion with $0.05 \%$ collagenase type II (Sigma, U.S.A.), under agitation for $45 \mathrm{~min}$ at $37^{\circ} \mathrm{C}$, a filtration and centrifugation at $800 \mathrm{~g}$ was performed, and the stromal vascular fraction (SVF) obtained. Afterward, the pellet (SVF) was resuspended with red blood cell lysis buffer ( $155 \mathrm{mM}$ of ammonium chloride, 12 $\mathrm{mM}$ of potassium bicarbonate and $0.1 \mathrm{M}$ of ethylenediaminetetraacetic acid, all from Sigma-Aldrich, Germany (in distilled water), incubated for $10 \mathrm{~min}$ at room temperature and centrifuged at $300 \mathrm{~g}$ for $5 \mathrm{~min}$. The supernatant was then discarded and the cell pellet was resuspended in $\alpha$-MEM medium (Invitrogen, U.S.A.), supplemented with $10 \%$ fetal bovine serum (FBS; Invitrogen, U.S.A.), and $1 \%$ antibiotic/antimycotic (Invitrogen, U.S.A.). Culture medium was changed $48 \mathrm{~h}$ after initial plating and every 3 days thereafter. hASCs were selected by plastic adherence and harvested (P0) 5 days after isolation at $90 \%$ confluence.

2.2. Flow Cytometry. hASCs (P3) were resuspended in a 3\% BSA (Sigma-Aldrich, France) solution and incubated for $20 \mathrm{~min}$ at room temperature, following manufacturer-recommended concentrations, with mouse antihuman antibodies CD105-FITC, CD73-PE, CD90APC, CD45-FITC, CD34-PE, and CD31-APC (BD Biosciences, Germany). Cells were subsequently washed with phosphate-buffered saline (PBS; Sigma, U.S.A.), centrifuged, fixed in 1\% paraformaldehyde (Sigma, U.S.A.), and analyzed on a FACs Calibur Flow Cytometer (BD Biosciences, U.S.A.) using the CELLQuest software V3.3.

2.3. In Vitro Creation of hASCs-Stacked CS Constructs. hASCs $\left(3 \times 10^{5}\right.$ cells $\left./ \mathrm{cm}^{2}\right)$ were plated in UP cell $35 \mathrm{~mm}$ thermoresponsive dishes (Nunc, Thermo Scientific, Denmark) and in six-well plates (BD Biosciences, U.S.A.), and cultured for 5 days in $\alpha$-MEM medium further supplemented with ascorbic acid (Sigma, Germany) at a concentration of $50 \mu \mathrm{g} / \mathrm{mL}$ (Figure 1A). CS were

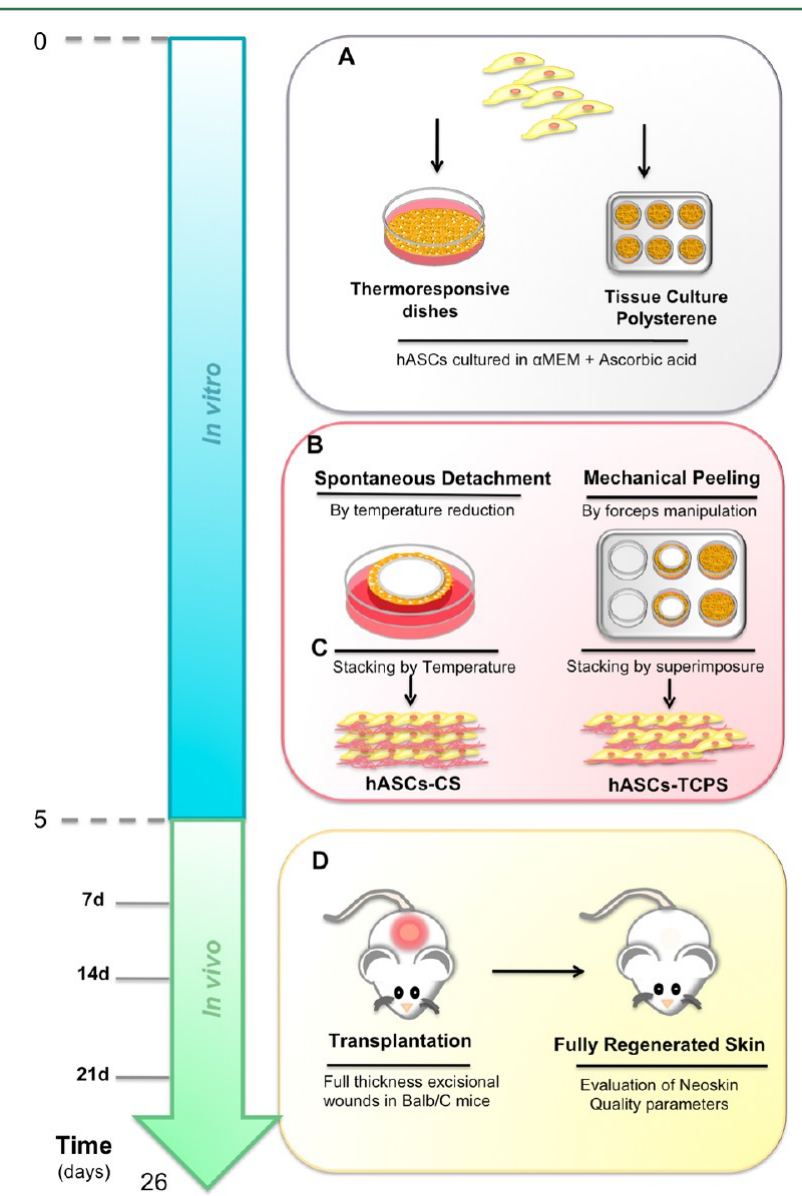

Figure 1. Schematic overview of the overall methodology followed along the work. (A) hASCs were seeded both in thermoresponsive dishes and standard tissue culture polystyrene (TCPS) plates, and cultured for 5 days in the presence of ascorbic acid. (B) Cell sheets (CS) were detached by temperature reduction and mechanical peeling and (C) superimposed to form 3D constructs, respectively hASCs-CS and hASCs-TCPS. (D) Constructs were transplanted into fullthickness excisional wounds created in the back of Balb/c mice and explants were retrieved after 7,14 , and 21 days to assess skin tissue regeneration.

retrieved, either by temperature-decrease from the thermoresponsive dishes, ${ }^{29}$ or by mechanical manipulation from the tissue culture polystyrene (TCPS) surfaces (Figure 1B). In brief, culture medium was removed from the culture dishes and replaced with $100 \mu \mathrm{L}$ of PBS. A poly(vinylidene difluoride) (PVDF; Immobilon-P, DURAPORE; Millipore Corporation, U.S.A.) membrane was placed over the cells in the thermoresponsive dishes, which were incubated at room temperature for $20 \mathrm{~min}^{30}$ Meanwhile, almost all the medium covering hASCs of a second thermoresponsive dish and the first hASCs-CS spontaneously detached was placed on the top of the second and the 
Table 1. Sequences of the Specific Mouse KGF, GAPDH, and ACTB Primers and Respective Resultant Amplicon Sizes and Annealing Temperatures Used in Real-Time PCR for Amplification

\begin{tabular}{clc} 
gene and accession number & \multicolumn{1}{c}{ sequence $\left(5^{\prime}-3^{\prime}\right)$} & amplicon size $(\mathrm{bp})$ \\
mouse GAPDH NM_008084.2 & GAAGGGCTCATGACCACAGT & 114 \\
mouse ACTB NM_007393.3 & TGCAGGGATGATGTTCTGGG & 183 \\
mouse KGF NM_008008.4 & GATCAAGATCATTGCTCCTCCTG & 50 \\
& AGGGTGTAAAACGCAGCTCA & 71
\end{tabular}

construct incubated for 20 minutes at $37{ }^{\circ} \mathrm{C}$ to promote $\mathrm{CS}$ adherence. The procedure was then repeated with a third CS in order to achieve a stable hASCs-CS engineered construct comprised by 3 CS. For the constructs of hASCs cultured in TCPS (hASCs-TCPS), the procedure was similar, but instead of temperature manipulation, forceps were used for mechanical pealing, manipulation and superimposure (Figure 1C).

2.4. Transplantation into Mice Full-Thickness Excision Wound. In vivo experimental protocol was approved by the Direcção-Geral de Alimentação e Veterinária (DGAV), the Portuguese National Authority for Animal Health, and all the surgical and necropsy procedures were performed according to the applicable national regulations respecting international animal welfare rules. A total of 36 animals (male, 5 weeks old Balb/c mice, Charles River Laboratories, France) were randomly divided into three groups: hASCs-CS, hASCs-TCPS, and the control group with no implant. Four animals were used per condition and per time point $(7,14$, and 21 days). Mice were anaesthetised with a mixture of Imalgene (ketamine; $75 \mathrm{mg} / \mathrm{kg}$ ) (Merial Portuguesa, Portugal) plus Domitor (medetomidine; $1 \mathrm{mg} / \mathrm{kg}$; Esteve Farma, LDA, Portugal), and skin was then shaved and epilated. A $1 \mathrm{~cm}$ diameter full-thickness excision was performed in each mouse at approximately $0.5 \mathrm{~cm}$ caudal to the intrascapular region. After placing 3D CS constructs, wounds were covered with transparent dressing Hydrofilm (Hartmann, U.K.) and a final set of bandages was used in order to avoid the dislocation of the transparent dressing and to protect the whole treatment set. Control wounds left empty were dressed likewise (Figure 1D). A subcutaneous injection of Depomedrol $(20 \mathrm{mg} / \mathrm{kg}$ BW; Pfizer, Portugal) was applied to the animals at days 0,7 , and 14 in order to delay wound healing. ${ }^{36}$ The animals were kept separately and, at the established end points, were euthanized by $\mathrm{CO}_{2}$ inhalation, and explants processed for histological analysis.

2.5. Standard Histology. The in vitro created hASCs-stacked CS constructs and skin explants were collected at days 3, 7, 14, and 21, and fixed in $10 \%$ formalin and paraffin embedded. Representative sections were stained with routine protocols of H\&E and Masson's trichrome. Sections were analyzed with a Axioplan Imager $\mathrm{Z1m}$ microscope (Zeiss, Germany), and images acquired and processed with AxioVision V.4.8 software (Zeiss, Germany).

2.6. Immunolabeling. Rabbit-antihuman/mouse keratin 14 (1:800; Covance, U.S.A.), collagen I (abcam, U.K.), and anti- $\alpha$ smooth muscle actin (1:100; Abcam, U.K.) were the primary antibodies used to characterize the explants. Alexafluor 488 donkey antirabbit (1:500, Invitrogen, U.S.A.) was applied as a secondary antibody for the keratin 14 and $\alpha$-smooth muscle actin detection, while for detection of collagen I, VECTASTAIN Elite ABC Kit (Vector Laboratories, U.S.A.) was used. Samples were examined with Axioplan Imager $\mathrm{Z} 1 \mathrm{~m}$ microscope and images were acquired and processed with AxioVision V.4.8 software.

2.7. Chromogenic In Situ Hybridization (CISH). Histological sections were deparaffinized, dehydrated, air-dried, and heated in a boiling water bath at $95{ }^{\circ} \mathrm{C}$ in pretreatment $1 \%$ MES buffer (Sigma, U.S.A.) for $10 \mathrm{~min}$. Enzymatic digestion was performed using $4 \mathrm{mg} /$ $\mathrm{mL}$ pepsin buffer (Worthington, U.S.A.) for $40 \mathrm{~min}$ at $37^{\circ} \mathrm{C}$ in a humid chamber. After dehydration, histological slides were air-dried, and positive DNA-biotin labeled probe (Pan Path, Netherlands) was applied. Denaturation was performed at $95{ }^{\circ} \mathrm{C}$ for $5 \mathrm{~min}$, and hybridization was achieved at $37{ }^{\circ} \mathrm{C}$ overnight. After that, samples were incubated for $10 \mathrm{~min}$ at $37^{\circ} \mathrm{C}$ with a stringency wash buffer (Pan Path, Netherlands). Streptavidin-peroxidase complex (Vector Laboratories, U.S.A.) was used for detection and DAB chromogen substrate system (Vector Laboratories, U.S.A.) for revelation. Mayer's hematoxylin was used for counterstaining.

2.8. Collagen Quantification. Collagen content of hASCS-CS and hASCs-TCPS in vitro constructs was quantified using the Sircol collagen assay kit (Biocolor, U.K.). The quantification was carried out in the $3 \mathrm{D}$ constructs fixed with formalin overnight at $4{ }^{\circ} \mathrm{C}$, according to the manufacturer's instructions. Briefly, constructs were homogenized in $500 \mu \mathrm{L}$ of extraction buffer and incubated overnight at $4{ }^{\circ} \mathrm{C}$ under stirring. Homogenates were then spun at $12000 \mathrm{rpm}$ for $60 \mathrm{~min}$ at $4{ }^{\circ} \mathrm{C}$ and pellets were then assayed for total collagen levels by comparison with a standard curve. Optical density was read at $540 \mathrm{~nm}$ in a Synergy HT microplate reader (Biotek, U.S.A.).

2.9. Real Time Reverse-Transcription PCR. Total RNA was extracted from in vivo skin explants using TRI reagent (Sigma, Germany) according to the manufacturer's instructions RNA quantity and purity were assessed with a NanoDrop ND-1000 spectrophotometer (NanoDrop Technologies, U.S.A.). Samples with a $260 / 280$ ratio between 1.6 and 2.0 were used for cDNA synthesis with qScript cDNA Synthesis kit (Quanta Biosciences, U.S.A.), according to the manufacturer's instructions in a MiniOpticon Real-Time PCR Detection System (BioRad, U.S.A.). Real-time PCR was carried out using the Perfecta SYBR Green FastMix (Quanta Biosciences, U.S.A.) and primers for each gene (Table 1) to compare the mRNA levels of mouse Keratinocyte growth factor (KGF) To evaluate the specificity of the amplified PCR fragments, melting curves were obtained by an increase of temperature from 55 to $95{ }^{\circ} \mathrm{C}\left(0.033{ }^{\circ} \mathrm{C} / \mathrm{s}\right)$. KGF expression, normalized with the internal controls,mouse GAPDH and mouse beta-actin, was assessed using the mathematical model proposed by Pfaffl $\left(2^{-(\Delta \Delta C t)}\right)$.

2.10. Image Analysis. Image analysis for the quantification of wound closure, vessels density, diameter and epidermal thickness was performed using Image J image analysis software (wayne, Rasband, NIH, U.S.A.) under the conditions detailed in each of the subsections below.

2.10.1. Wound Closure. Planimetric digital images were taken on the day of surgery and at days, 7,14 , and 21 postimplantation for all four animals of each condition and time point. The percentage of wound closure was then calculated as

$$
\frac{\text { area of original wound }- \text { area of actual wound }}{\text { area of original wound }} \times 100
$$

Wound was considered completely closed when the actual wound area was equal to zero. ${ }^{32}$

2.10.2. Quantification of Vessels Density and Diameter. The number of vessels was quantified at days 14 and 21 postoperative in the $\alpha$-smooth muscle actin (SMA) stained samples. Quantification and measurement were performed in randomly selected five high - power fields of five nonconsecutive tissue sections per time point and per animal within each group. Only vessels with a diameter of $<50 \mu \mathrm{m}^{33}$ were considered. Mean results of the number of vessels per field are expressed as vessels density.

2.10.3. Epidermal Thickness Measurement. Epidermal thickness of the formed neoskin was evaluated on H\&E stained histological sections of both experimental and control groups explanted at day 21 
i)

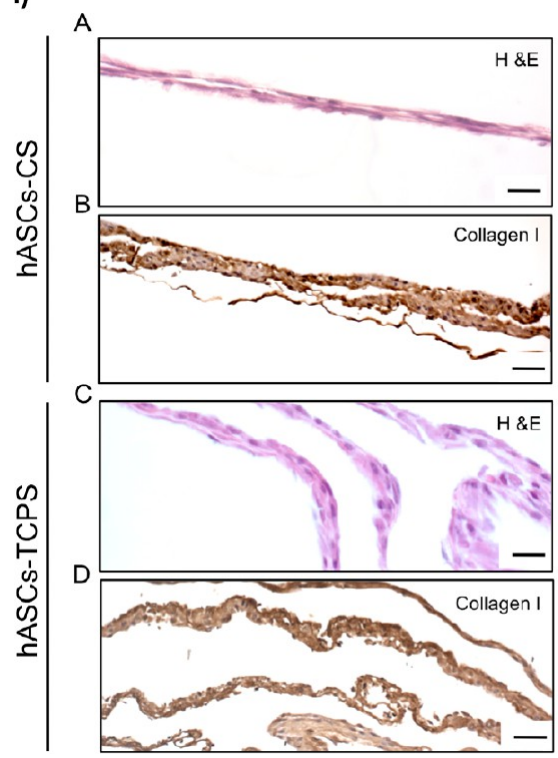

ii)

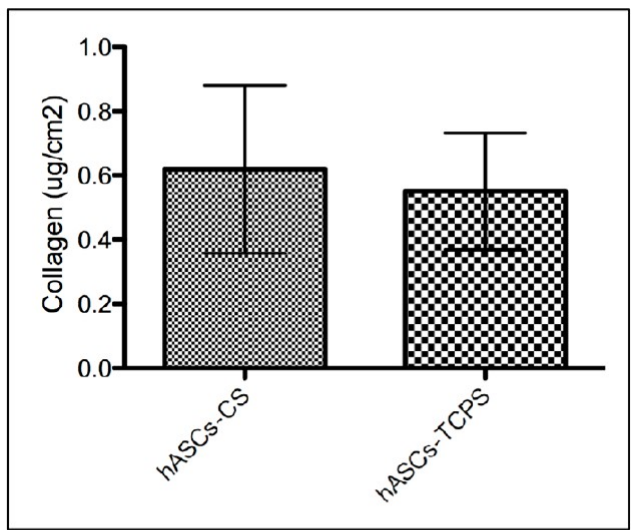

Figure 2. In vitro characterization of the hASCs cell sheets (CS) constructs. (i) Histological characterization of the 3D constructs formed by detachment by temperature reduction $(A, B)$ and by mechanical peeling $(C, D)$; distinct stability of the constructs is underlined. The nature of the deposited extracellular matrix was evidenced after imunostaining the $3 \mathrm{D}$ constructs for collagen type I (B, D) that was (ii) further quantified by Sircol collagen assay, expressed by $\mu \mathrm{g} / \mathrm{cm}^{2}$ of each construct.

and based in the methodology described by Larouche et al. ${ }^{34}$ Four tissue sections per animal within each group were analyzed by randomly selecting five high-power fields in each section and performing five measurements of the epidermal thickness per field.

2.10.4. Skin Maturity Quantification. The quality of neoskin formed was evaluated after 21 days of implantation, by quantifying the epithelial maturation and dermal differentiation, on $H \& E$ and Masson's Trichrome stained histological samples, respectively, according to criteria described by Sun et al. ${ }^{23}$ In more detail, for epithelial maturation, the grading was defined as follows: grade 1 , thin and with no reticulation; grade 2, occasional reticulation; grade 3 , moderate reticulation; and grade 4, thick and with complex reticulation. The grading for dermal differentiation was as follows: grade 1, thin, dense, and monotonous fibrosis; grade 2, thicker but still dense and monotonous fibrosis; grade 3 , two layers but not completely discrete; grade 4, two discrete layers with superficial fibrosis and loose alveolar tissue within the deep layer.

2.11. Statistical Analysis. Statistical analysis of wound closure and real-time RT-PCR was performed using two-way ANOVA with Bonferroni post tests. Data of vessels number at day 14 and diameter for both days 14 and 21, were analyzed by the nonparametric Kruskal-Wallis test, with Dunns post test. Data analysis of vessel numbers at day 21, epidermal thickness, epithelial maturation and dermal differentiation was carried out by one-way ANOVA with Tukey's post tests (GraphPad Prism 4.02). Significance levels, determined using post tests between groups were set at $*_{p}<0.05$, $* * p<0.01$, and $* * * p<0.001$.

\section{RESULTS}

3.1. hASCs-CS and hASCs-TCPS Constructs Nature. The mesenchymal nature of the isolated hASCs was confirmed prior creating the CS, showing to meet the standards established by the International Society for Cellular Therapy ${ }^{35}$ (Figure S1).

To assess the feasibility and the success of the proposed methodologies to obtain hASCs CS as well as the respective constructs composed by three stacked CS, in vitro characterization was performed based on H\&E staining. The adhesive nature of the generated hASCs-CS was clearly confirmed after stacking and building up the $3 \mathrm{D}$ constructs (Figure $2 \mathrm{i} ; \mathrm{A}, \mathrm{B}$ ). A consistent and easily manageable construct was obtained in contrast to the hASCs-TCPS stacked CS that formed an unstable construct upon manipulation (Figure 2i;C,D). Despite the differences regarding the CS retrieval methodology, no significant differences on the collagen content were observed between the hASCs-CS and hASCs-TCPS constructs (Figure 2ii).

3.2. In Vivo Performance. 3.2.1. Transplanted hASCs Constructs Showed No Effect in Wound Closure. The tested conditions had no significant effect over wound closure (Figure $3 \mathrm{i}, \mathrm{ii})$. The percentage of wound closure did not vary between the test groups, including the control, and along the implantation time. Wounds were progressively closing, reaching total closure at day 21 postoperative for all the conditions. Both the macroscopic images of the wounds (Figure $3 i$ ) and the wound closure measurements (Figure 3ii) confirmed this similarity among the test groups. Wound progression was analyzed after H\&E staining (Figure 3iii), confirming that the major differences were noticed at the end point. hASCs-CS lead to not only full re-epithelialized wounds but also to the formation of appendages in the neoformed matrix.

3.2.2. Transplanted hASCs Enrol Neodermis Formation. The contribution of the transplanted hASCs as elements of the newly formed tissue was assessed by CISH technique (Figure 4). At days 3 and 14, the transplanted hASCs were mostly localized at wound margins (Figure 4A,C), whereas at day 21, they were integrating the matrix of neoformed tissue (Figure 4B). The number of human cells appeared to decrease with time, which was highly notorious from day 14 to day 21 . At the end point, hASCs were still visible in the neoformed tissue, being exclusively present within the dermal matrix and surrounded by the host cells, only with the nuclei stained (Figure 4D). In fact, at the latest time point, hASCs did not seem to integrate other relevant tissue structures such as vessels or epidermis. The transplanted hASCs also seemed to impact dermal degree of maturation (Figure 5). Although not statistically different (Figure 5i), the dermal component of 
A

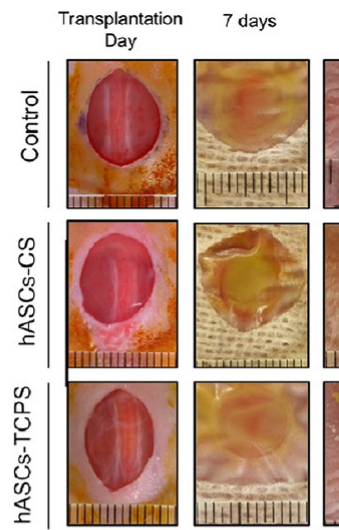

c
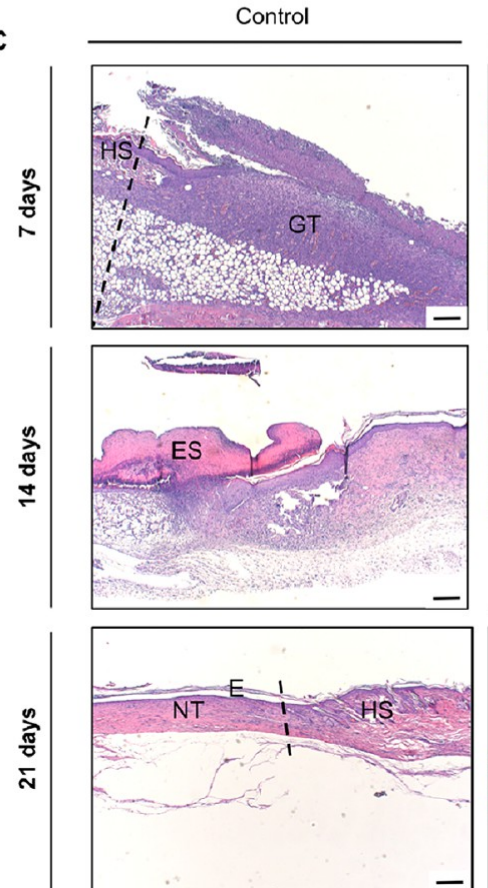

21 days
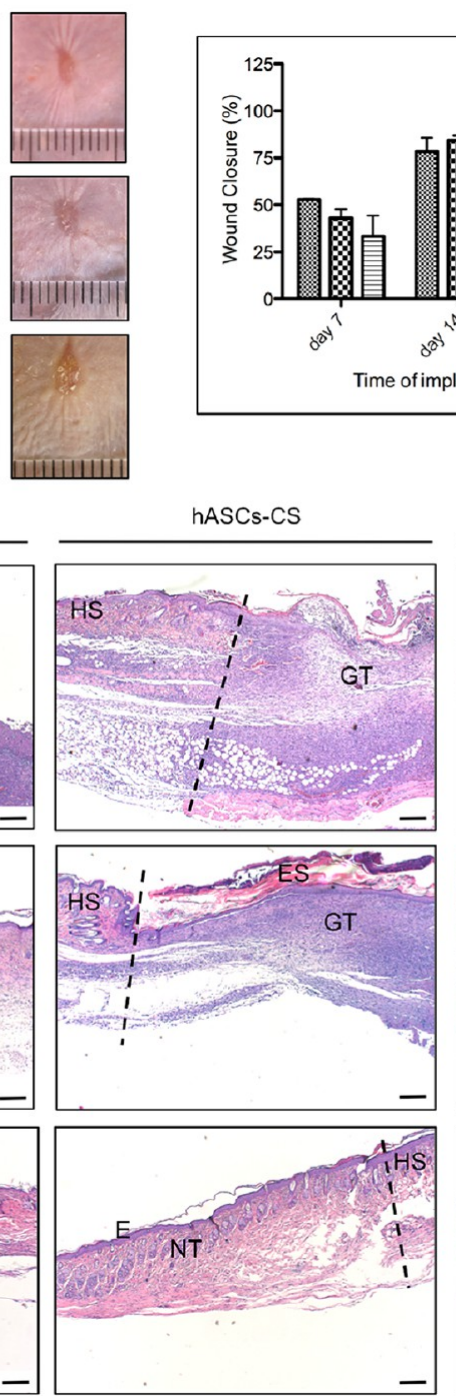
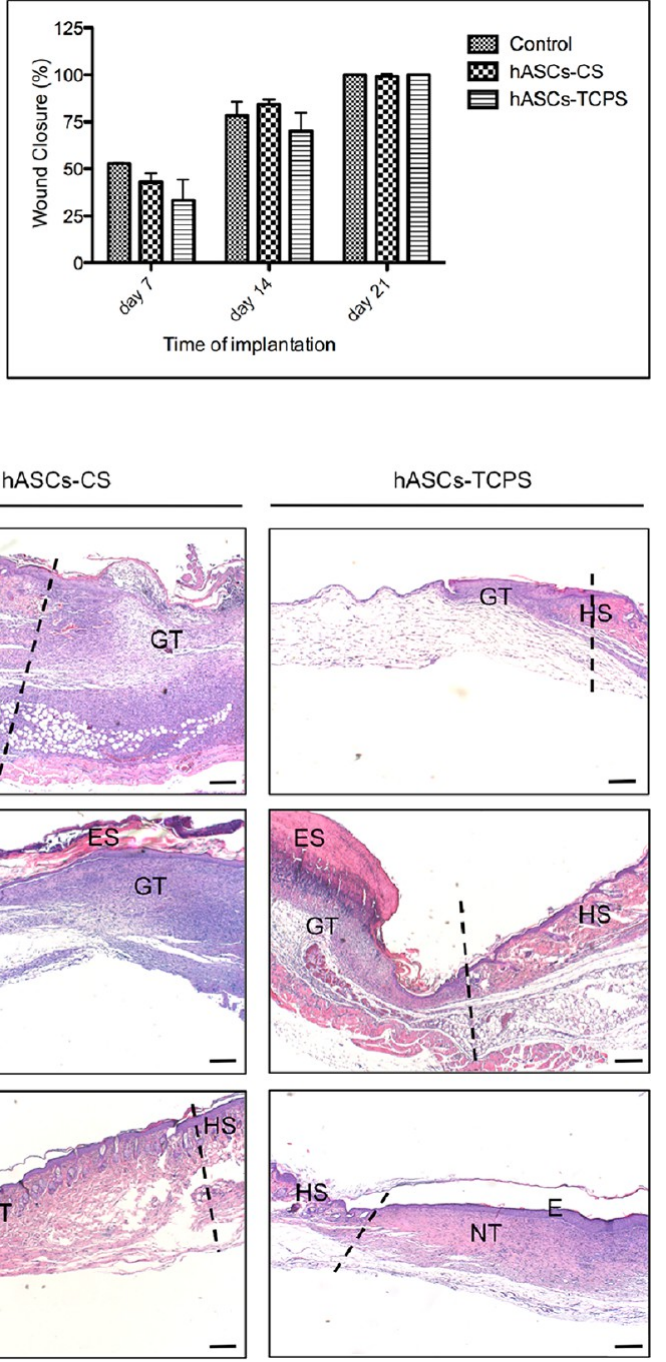

Figure 3. Effect of hASCs-CS and hASCs-TCPS 3D constructs over wound closure. (A) Representative macroscopic images of the different experimental groups wounds, at the time of transplantation and after 7, 14, and 21 days. (B) Representation of the percentage of wound closure in the different experimental groups along the implantation time. (E) Illustrative images of H\&E-stained histological sections of explants at day 7, 14, and 21, highlighting the wound healing progression along the time frame chosen. Scale bar corresponds to $200 \mu \mathrm{m}$ and GT, granulation tissue; ES, eschar; HS, healthy skin; NT, neotissue; and E, epithelium. Wound margins were delineated.

the hASCS 3D constructs conditions (Figure 5ii;B,C) appeared to have a higher differentiation degree than the dermal tissue formed in the control condition (Figure 5ii;A).

3.2.3. hASCs-CS and hASCs-TCPS Promote Neotissue Vascularization. The influence of the proposed strategies over neovasculature formation was analyzed based on vessels density and diameter, at days 14 and 21, quantified after SMA staining (Figure 6ii). At day 14, the density of vessels within each group varied (Figure 6i;A). The control values ranged from 4 to 55, while for the experimental conditions data varied within narrow intervals. Nonetheless, although having distinct values for the minimum and maximum, the median value was very similar between the groups. The observed tendency within each group at day 14 changed at day 21 (Figure 6i;B); the density of vessels in the control and the hASCs-CS were not so diverse, but appeared to be different from the hASCs-TCPS group. The median vessel density values successively increased from the control $($ median $=8.5)$ to the hASCs-CS $($ median $=$
15.5) and hASCs-TCPS (median = 21.5) treatment groups (Figure 6i;B).

A distinct tendency was observed regarding the vessel diameter (Figure 6i;C,D). A similar distribution of the data was observed at day 14 for all the test groups however, median vessel diameter value for the hASCs-TCPS group (median = 17.7) was significantly higher than for control (median = 15.2; $p<0.05$ ) and hASCs-CS (median $=15.1 ; p<0.001$ ) groups (Figure $6 \mathrm{i} ; \mathrm{C}$ ). On the contrary, at day 21 the median vessels diameter for the hASCs-TCPS group (median = 11.75) was significantly lower $(p<0.001)$ than for control (median = 21.22) and hASCs-CS (median = 16.47) groups (Figure 6i;D).

3.2.4. Epidermal Morphogenesis is Modulated by hASCsCS. Re-epithelization is a fundamental achievement in wound healing therefore, morphogenesis and quality of the epidermis formed in each test group was analyzed. From the H\&E images, it was clear that the newly formed epidermis is different from group to group (Figure 7i). Epidermis of the hASCs-CS group 

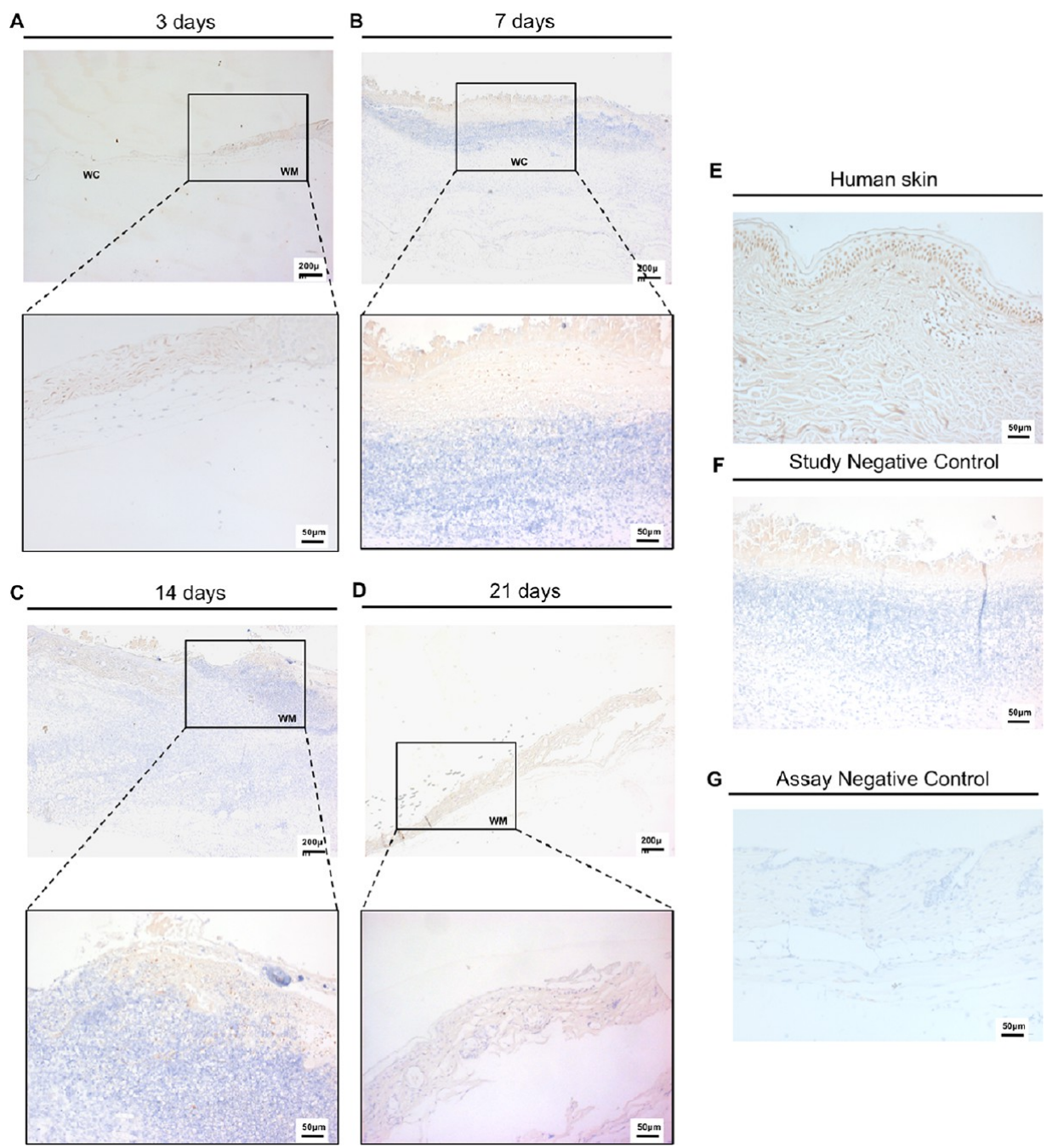

Figure 4. Detection of human cells on mice histological sections of correspondent explants at (A) day 3, (B) day 7, (C) day 14, and (D) day 21 postoperative by $\mathrm{CISH}$. (E) Positive control and (F, G) negative controls are also shown. Nuclei were stained with hematoxylin and scale bars correspond to $200 \mu \mathrm{m}$ or $50 \mu \mathrm{m}$ : WC, wound center; WM, wound margin.

was significantly thicker than the epidermis of control $(p<$ $0.01)$ and hASCs-TCPS $(p<0.05)$ groups (Figure 7iv;B).

Additionally, the expression pattern of keratin 14 was also found to be different between the groups. In the control group, the expression of keratin 14 was restricted to epidermal basal cells (Figure 7ii;A), while for both hASCs-CS and hASCsTCPS groups, its expression was located in both basal and suprabasal layers (Figure 7ii; B,C). Nonetheless, rete ridges-like structures, clearly identified after H\&E staining (Figure 7iii;A) and keratin 14 labeling (Figure 7iii;B) were only detected in hASCs-CS treatment group. Furthermore, a significant number of hair follicles were present in the hASCs-CS treated group (Figure 7ii;B) and the epithelial maturation grading assessment (Figure 7iv;A) revealed a significantly higher epithelial maturation degree exhibited by the experimental groups in comparison to the control group.

Moreover, the assessment of mouse KGF mRNA (Figure 7v) revealed a significant upregulation $(p<0.05)$ of the expression of this gene for the hASCs-CS group at days 3 and 14 postoperative not only in comparison to control, but, more importantly, to the hASCs-TCPS group.

\section{DISCUSSION}

A scaffold-free CS engineering-based approach that take advantage of cellular mechanisms, as traditional cell-based therapies, but that overcomes the shortcoming of poor cell engraftment, impacting full-thickness skin wound regeneration is herein proposed.

Stacked hASCs CS-based constructs were created using thermoresponsive and standard TCPS cell culture surfaces. The main advantage of using CS engineering technology based on thermosensitive reactions refers to the CS natural adhesive nature that allow not only an almost immediate interaction with the host tissues but also the possibility to build stable 3D constructs that can be easily manipulated, which might be related with a superior ECM organization promoted in those surfaces. By adding to the culture media ascorbic acid, implicated in the production and organization of extracellular matrix, namely, in stimulating collagen production, ${ }^{4,36}$ we were able to lead the secretion and organization of an abundant endogenous ECM and the formation of a confluent hASCs layer exhibiting a thick collageneous matrix within a 5 days period, independently of the culture surface. Nonetheless, despite the apparent robustness of the CS, the stability of the constructs built by CS stacking varied with the surfaces used to 

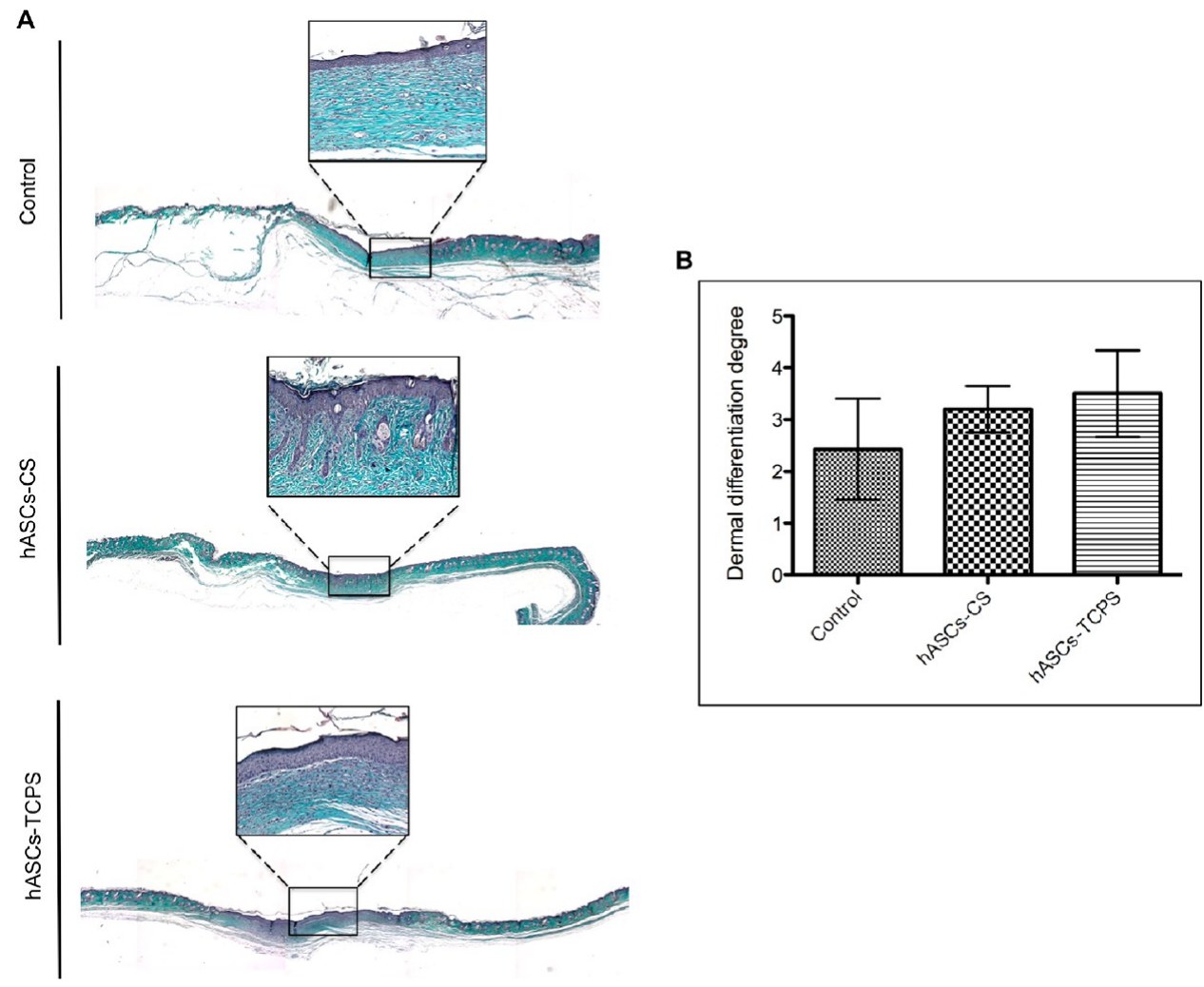

Figure 5. Neodermis quality evaluation. (A) Histological analysis of the 21 days explants of the control, hASCs-CS and hASCs-TCPs stained with Masson Trichrome, evidencing in higher maginification pictures the differences in the dermal matrix organization among the groups. (B) Representation of the dermal degree of differentiation obtained after a graded qualitative.

obtain the CS. Previous studies ${ }^{4,37}$ correlated the time required to retrieve handlabeled CS, normally around 28 days, depending on the ECM deposition rate, with their superimposing capacity, which might be the reason for the instability observed for the hASCs-TCPS constructs. Rodent and human skin have major dissimilarities; the natural contraction in rodents, namely, in mouse models due to the existence of paniculosus carnosus layer undertheath the skin, and the healing in humans via re-epithelialization and granulation tissue formation $^{38}$ are among them. Thus, rodent skin wound models despite their valuable contribution regarding skin healing, have been updated in order to minimize the limitations associated to those differences. ${ }^{38}$ In this study a steroid (depomedrol) was administrated to attenuate wound contraction ${ }^{39}$ and also at delaying the healing process. ${ }^{31}$ Although considerable contraction was observed, it seems to be similar for all the conditions, thus, we consider that this factor did not compromise the direct comparison between the test groups. In fact, the absence of differences regarding wound closure between the control and experimental hASCs-CS groups, all achieving complete closure at day 21 , are in agreement with another work with a mice model with silicone splint fixed on the skin to prevent wound contraction. ${ }^{14}$ Based on this, it might be speculated that contraction was not the predominant healing variable and that the transplanted hASCs have an effect on other wound healing features. The improved effect of hASCs on cutaneous wound healing has been evidenced in the literature. ${ }^{3,12,40}$ Transplanted human-origin cells were identified within the restored skin and up to 21 days. This prolonged engrafment of hASCs is in accordance with the findings of a recent study that similarly propose CS of hASCs to treat full- thickness wounds ${ }^{14}$ but has not been reported in other works, ${ }^{41,42}$ which seems to confirm our hypothesis that the transplantation of hASCs within their own ECM, as CS, improves cell engraftment. Nonetheless, transdifferentiation phenomena, particularly into the epidermal and vascular lineage, reported in other works, ${ }^{3-5,11,14,43}$ contrary to our expectation, was herein not detected. As stressed by others, ${ }^{44,45}$ the heterogeneous biochemical and paracrine effector functions are of major importance among mesenchymal cells role. Our findings suggest that the transplanted hASCs might be acting mainly through paracrine effects on the resident cells, ${ }^{9}$ therefore, targeting several aspects of regeneration, such as the extension of re-epithealization and angiogenesis. Nie et al. demonstrated that hASCs are capable of secreting angiogenic factors, including VEGF, HGF, and FGF2, in vitro and in vivo. ${ }^{5}$ Moreover, the secretion of angiogenic and antiapoptotic factors by hASCs was shown to affect endothelial cell behavior ${ }^{45}$ and, consequently, the newly formed vascular network and the overall vascularization of the neotissue. An impact of the transplanted hASCs over vessel density and diameter at the implantation site was clearly demonstrated in this work. Those parameters were assessed at days 14 and 21 postoperative, time points where inflammation is attenuated and in which cellular remodeling/maturation phase in wound healing is critical. ${ }^{18}$ While no major differences were observed at day 14, a higher density of vessels was detected in the experimental groups at day 21 , which is in agreement with previous works. ${ }^{11,14}$ Interestingly, a tendency of narrow vessel diameter intervals was observed for the experimental conditions after 21 days of implantation, which seems indicative of stabilization of the healing process. $^{46}$ In fact, a high degree of regeneration 
i)

A

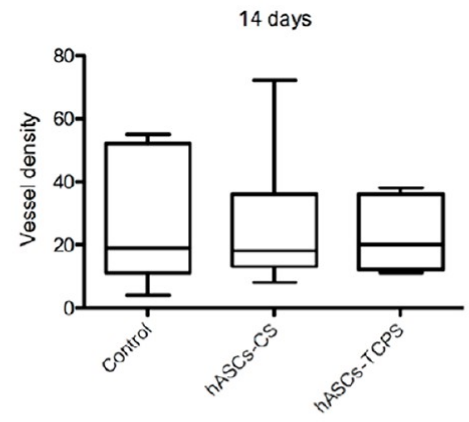

C

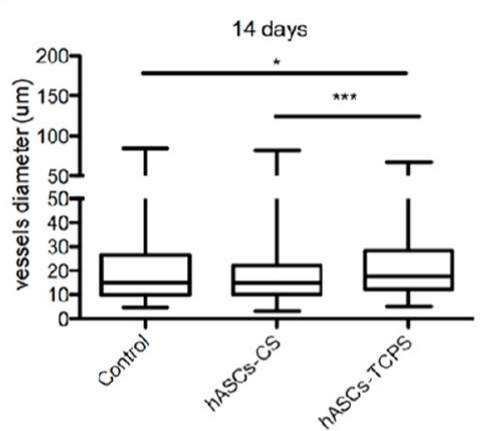

ii) $\mathrm{A}$

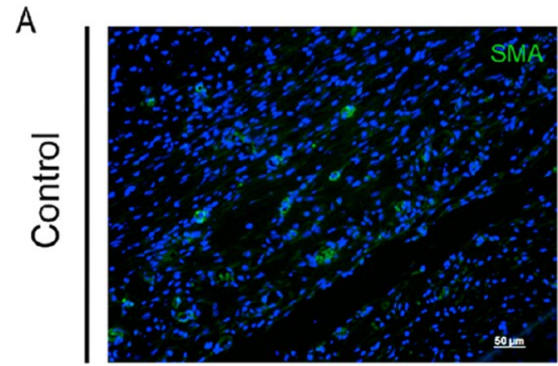

B

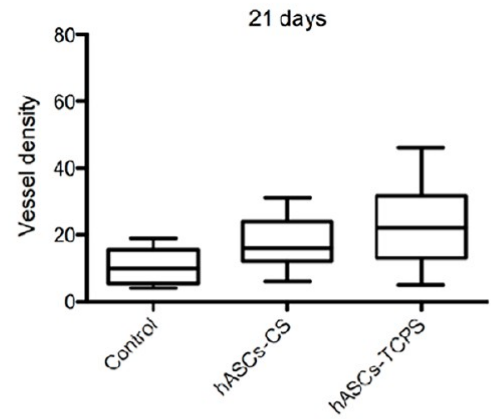

D

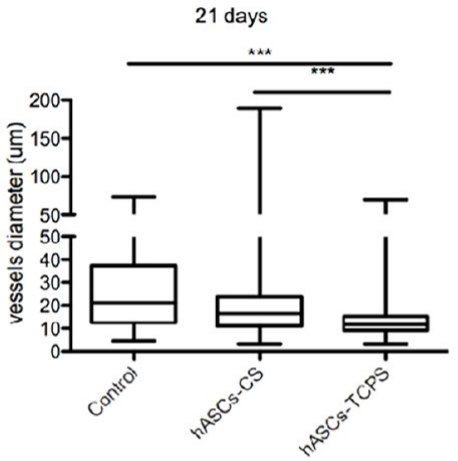

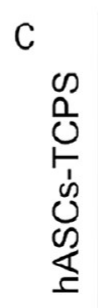
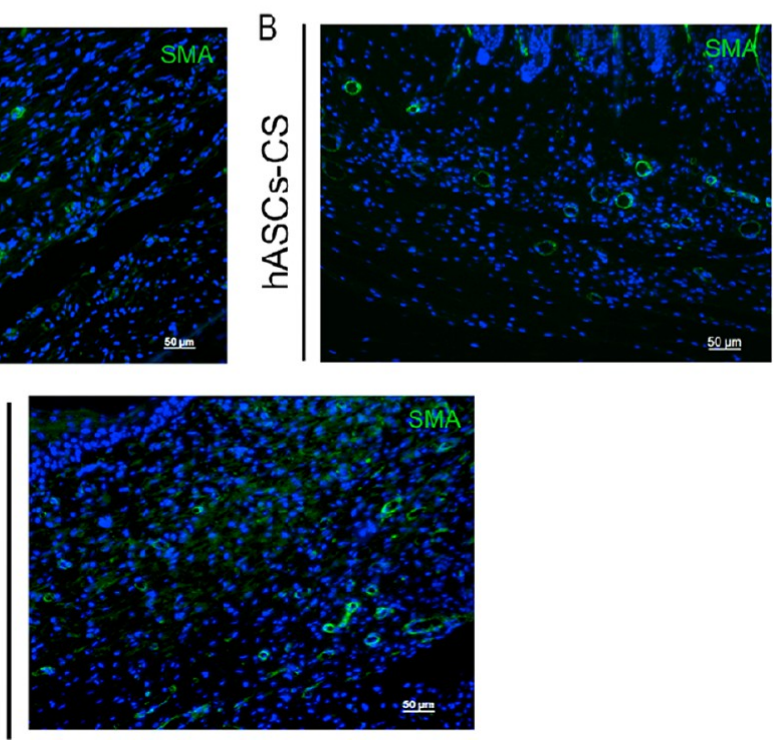

Figure 6. Neovascuarization effect of the proposed hASCs CS-based constructs. (i) Representation of (A, B) the density of vessels and of the (C, D) diameter of vessels, present in the wounded area after (A, C) 14 and (B, D) 21 days of implantation. Quantification was performed on the (ii) vessels positive for $\alpha$-smooth muscle actin (green) after immunohistochemical assay in histological samples of explants correspondent to (A) control, (B) hASCs-CS, and (C) hASCs-TCPS; $* p<0.05$ and $* * * p<0.001$.

translated by a well-organized dermal matrix with arranged dermal fibrils, a highly reticulated epidermis and more importantly the presence of new hair follicles was observed at day 21 for the hASCs-CS group. Furthermore, at day 21, the thicker epidermis observed in the hASCs-CS group as well as the distinct expression pattern of $\mathrm{k} 14$ in the wounded area treated with hASCs-CS and hASCs-TCPS gained our attention. While k14 expression in the control was restricted to the epidermal basal layer of cells, in the experimental groups k14 was displayed also in suprabasal layers, suggesting that suprabasal keratinocytes may retain the immature character of basal cells, ${ }^{46}$ although being part of a mature stratified epithelium that is being formed. Surprisingly, rete ridges-like structures were also identified in the neoepidermis of hASCsCS experimental group at day 21 . These structures are typical of human tissue, but normally are not present in mice. ${ }^{38}$ Actually, as discussed by Yannas et al., ${ }^{47}$ the presence of rete ridges in humans is one of the main features that distinguish intact and regenerated skin from scar. Knowing that the mesenchymal-epithelial cells interaction plays an essential role in organogenesis, ${ }^{48,49}$ we might assume that the observed rete ridges-like formation was a consequence of the distinct epidermal morphogenesis induced by the treatment with hASCs, although only for the hASCs-CS group. Evidences of 
i)

ii)
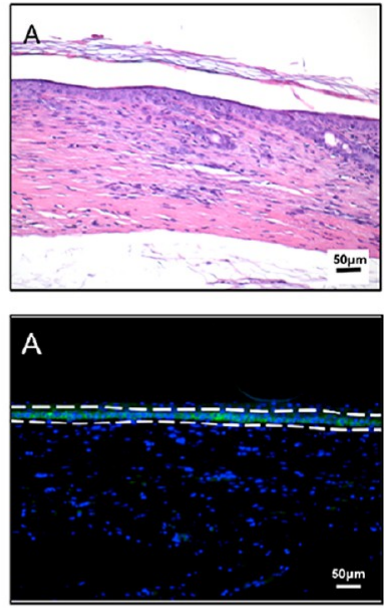
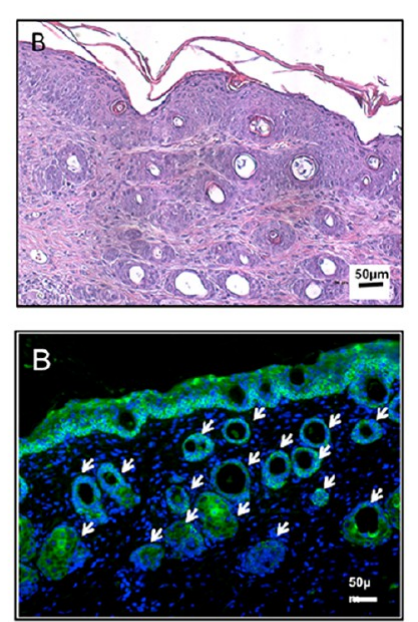
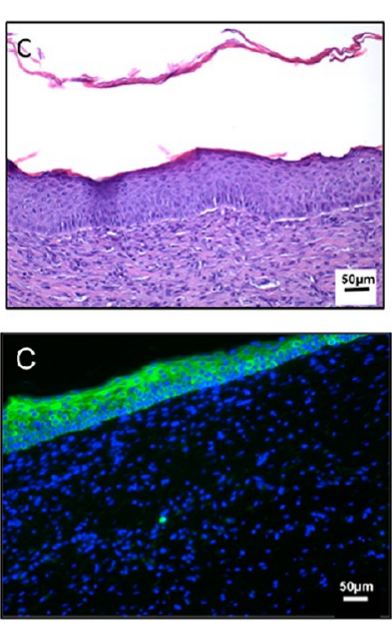

iii)
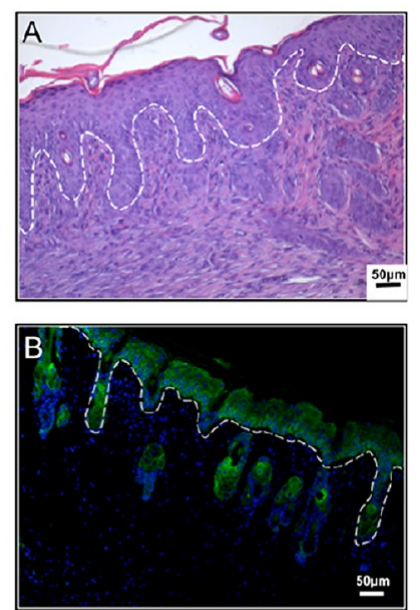

iv)

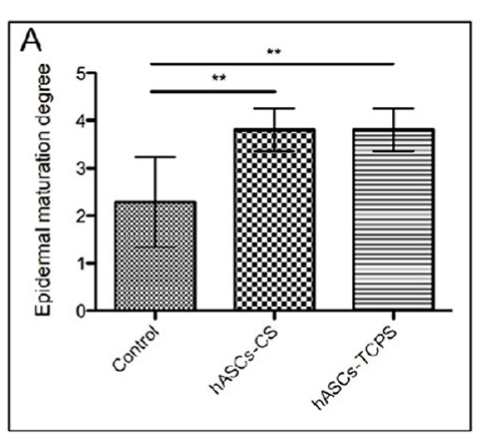

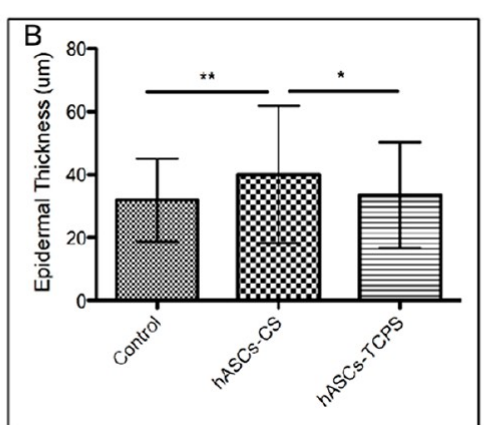

v)

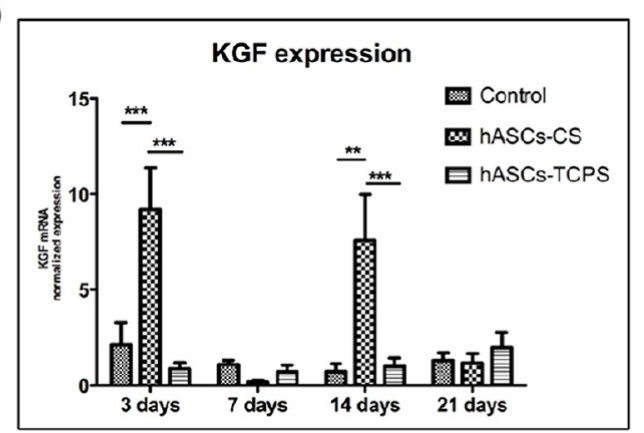

Figure 7. Epidermal morphogenesis. (i) Histological analysis of the 21 days explants of (A) control, (B) hASCs-CS, and (C) hASCs-TCPS groups stained with H\&E evidencing the differences in the thickness of the epidermis among the groups. (ii) Immunohistochemical analysis of the expression of keratin 14 (green). The differential keratin 14 pattern expression at day 21 seems indicative of a (A) distinct epidermal morphogenesis. In the control k14 was displayed just in the basal layer (limited by the dashed line), whereas in both hASCs-CS (B) and hASCs-TCPS (C) groups, k14 was present in the basal and suprabasal epidermal layers. In the hASCs-CS group, k14 was also expressed in the hair follicles (arrows), present in significant number in this experimental group. (iii) Rete Ridges-like structures (outlined by the dashed line) were identified in the neoepidermis of the hASCs-CS experimental group, at day 21. Those structures were clearly observed in the (A) H\&E stained samples and also after (B) immunohistochemical identification of the expression of keratin 14 (green) by the basal cells. DAPI (blue) was used as nuclear staining for the immunostaining. (iv) Representation of the (A) epidermal maturation degree obtained after a graded qualitative analysis of $\mathrm{H} \& \mathrm{E}$ histological tissue samples of the different experimental groups of day 21 and (B) measurements of the thickness of the epidermis for the different experimental groups. (v) Graphical representation of Real time RT-PCR analysis of KGF differential expression among the different groups at different time points of implantation: $* p<0.05, * * p<0.01, * * * p<0.001$. Scale bar corresponds to $50 \mu \mathrm{m}$.

the acceleration of epidermal growth by mesenchymal stem cells (MSCs) and of the role of these cells in inducing keratinocytes to reorganize rete ridge-like structures are reported. ${ }^{13}$ Although KGF is present at very low levels in homeostatic skin, its expression is highly upregulated within 24 $\mathrm{h}$ after injury, ${ }^{50}$ and therefore, its involvement in the formation of rete ridges-like structures might be expected. KGFexpressing keratinocytes are known to affect epidermal structure and morphology, while KGF induces proliferation of keratinocytes residing in the normally quiescent suprabasal layers of the epidermis. ${ }^{51}$ Moreover, a significant $k 14$ expression by cells in the suprabasal layers of regenerating epidermis, which is in agreement with our results, and a need for the direct mesenchymal-epithelial contact have been reported as essential for rete ridge-like structures replication. ${ }^{13}$ These facts, together with the upregulated expression of KGF in the hASCs-CS group at an early timepoint, support our hypothesis of the hASCs effect on the production of KGF that consequently modulates the phenotype of suprabasal cells influencing epidermal morphogenesis. In fact, the presence of rete ridgeslike structures was only observed in the hASCs-CS group, which can be justified by the almost immediate, stronger, and more prolonged interaction between the host cells and the transplanted cells of the hASCs-CS constructs due to their natural adhesive properties. Moreover, the instability of the hASCs-TCPS constructs probably results in their disaggregation into single CS after transplantation corroborating previous findings that have indicated that multilayered CS constructs have a superior outcome in terms of tissue formation, more complete regeneration and higher therapeutical effects in comparison to single CS. ${ }^{52-54}$ Although enhanced wound healing was also recently attributed to stacked hASCs-CS constructs in opposition to single hASCs cell sheets, ${ }^{14}$ comparable features in terms of vessels density, collagen deposition, and neotissue organization between the experimental groups do not corroborate the assumption. In that work, no considerations were made and, due to experimental ambiguities, cannot be taken regarding the nature of the CS ECM or the stability of the proposed hASCS constructs, which could be determinant for the obtained outcome. It is therefore our belief that the stability of the constructs, directly determined by the degree of cohesiveness of cell-cell and 
cell-ECM interactions, is determinant in differentiating the healing mechanisms and justifies the differences observed between the hASCs-TCPS and hASCs-CS groups regarding epidermal morphogenesis.

Based on our observations, and on previous and extensive knowledge reported for CS engineering we propose a model of the cellular interactions responsible for the superior epidermal morphogenesis that occurs after transplantation of hASCs-CS constructs in a full-thickness skin wound (Figure 8).

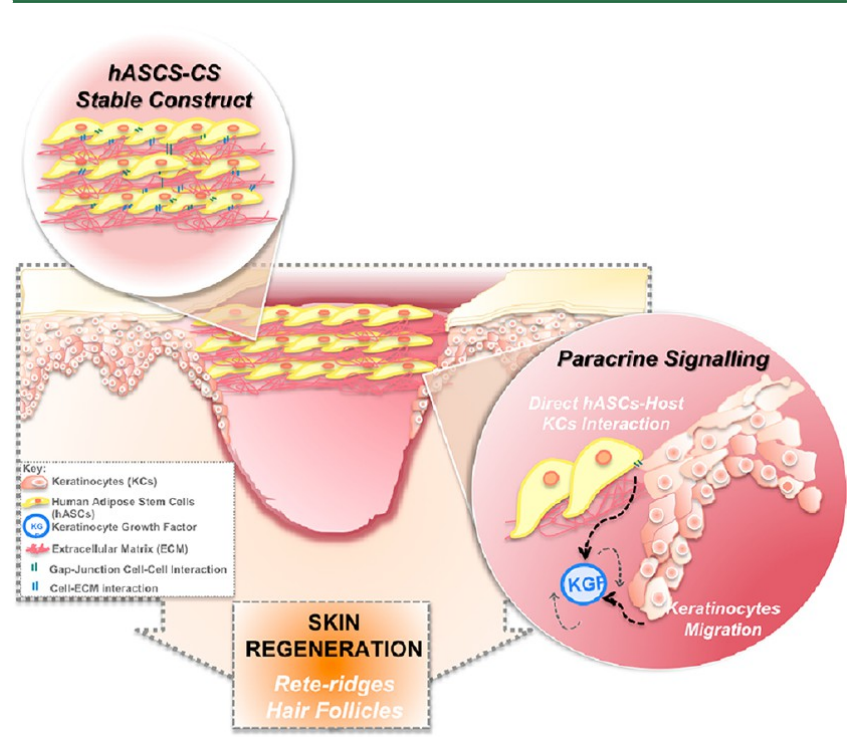

Figure 8. Schematic representation of the proposed paracrine signaling, between mesenchymal and epithelial cells, involved in the observed neo-epidermis morphogenesis. The early direct interaction of transplanted hASCs-CS with resident cells induces KGF secretion by hASCs. Knowing that KGF expression is upregulated after injury promoting proliferation and migration of keratinocytes residing in the normally quiescent suprabasal layers of the epidermis, a paracrine KGF signaling loop is established. It is therefore proposed that KGFexpressing keratinocytes strongly influences the rete-ridges and hairfollicles formation, as a consequence of KGF involvement in the epidermal morphogenesis.

The hASCS of the superimposed CS, embedded within their own collagenous matrix, communicate between them through gap junctions and through the deposited ECM forming extremely stable constructs. Upon transplantation into the wound bed, it is likely that similar communication levels between the transplanted hASCs and the resident cells occur. Direct mesenchymal-epithelial interaction affects the expression of KGF by keratinocytes and by MSCs. While KGF is highly upregulated within $24 \mathrm{~h}$ after injury, ${ }^{50}$ inducing the proliferation of keratinocytes residing in the normally quiescent suprabasal layers of the epidermis, ${ }^{51}$ KGF is expressed by MSCs only under mesenchymal cell-keratinocyte direct interaction. ${ }^{13}$ Therefore, the natural adhesive character of the created constructs promote the direct hASCs-resident keratinocytes interaction upregulating KGF expression and consequently an enhanced proliferation of K14 positive cells, identified in the basal and suprabasal layers of the regenerating epidermis at late time points. Thus, we suggest that hASCs-keratinocytes contact-dependent paracrine pathways mediate and are likely to predominantly regulate specific events such as the formation of rete ridges-like structures during epidermal regeneration.

\section{CONCLUSION}

This study provides evidence that hASCs CS constructs produced in thermosensitive surfaces could be fabricated within a short time frame, yet showing a greater stability than hASCsTCPS. Upon transplantation into full-thickness excisional wounds, both strategies lead to a prolonged engraftment of hASCs, to a stabilized neovascularization, dermal remodeling, and surprisingly, a superior epidermal morphogenesis, though transdifferention of the engrafted cells was not observed. hASCs-CS group re-epithelization was characterized by a high number of hair follicles, presence of rete ridge-like structures, and yet by a higher degree of epidermal maturation. Our findings further suggest that the transplanted hASCs might be acting through paracrine effects with the resident cells, thus, influencing the different aspects of skin regeneration.

The simplicity of the methodology used, the short time frame of hASCs-CS production, and the significant impact over full-thickness excisional wounds regeneration reinforce the potentialities of this strategy to be particularly advantageous for cell transplantation in a clinical scenario.

\section{ASSOCIATED CONTENT}

\section{S Supporting Information}

The video shows the retrieval of the hASCs cell sheets from the thermoresponsive dishes, confirming the robustness of the retrieved CS by temperature reduction, allowing its manipulation, than can be further facilitated by the use of PDVF membranes, as it was done for the in vivo transplantation and in vitro CS stacking to form the $3 \mathrm{D}$ constructs. This material is available free of charge via the Internet at http://pubs.acs.org.

\section{AUTHOR INFORMATION}

\section{Corresponding Author}

*E-mail: apmarques@dep.uminho.pt. Tel.: 00351510906.

\section{Notes}

The authors declare no competing financial interest.

\section{ACKNOWLEDGMENTS}

We would like to thank Hospital da Prelada (Porto), in particular, to Dr. Paulo Costa for the lipoaspirates collection and for financial support by Skingineering (PTDC/SAU-OSM/ 099422/2008), Portuguese Foundation for Science and Technology (FCT) funded project. The research leading to these results has also received funding from the European Union's Seventh Framework Programme (FP7/2007-2013) under Grant Agreement No. REGPOT-CT2012-316331POLARIS.

\section{REFERENCES}

(1) Martin, P. Wound healing-aiming for perfect skin regeneration. Science 1997, 276 (5309), 75-81.

(2) Singer, A. J.; Clark, R. A. Cutaneous wound healing. N. Engl. J. Med. 1999, 341 (10), 738-46.

(3) Altman, A. M.; Matthias, N.; Yan, Y.; Song, Y. H.; Bai, X.; Chiu, E. S.; Slakey, D. P.; Alt, E. U. Dermal matrix as a carrier for in vivo delivery of human adipose-derived stem cells. Biomaterials 2008, 29 (10), 1431-42.

(4) Trottier, V.; Marceau-Fortier, G.; Germain, L.; Vincent, C.; Fradette, J. IFATS collection: Using human adipose-derived stem/ stromal cells for the production of new skin substitutes. Stem Cells 2008, 26 (10), 2713-23.

(5) Nie, C.; Yang, D.; Xu, J.; Si, Z.; Jin, X.; Zhang, J. Locally administered adipose-derived stem cells accelerate wound healing 
through differentiation and vasculogenesis. Cell Transplant 2011, 20 (2), 205-16.

(6) Liu, P.; Deng, Z.; Han, S.; Liu, T.; Wen, N.; Lu, W.; Geng, X.; Huang, S.; Jin, Y. Tissue-engineered skin containing mesenchymal stem cells improves burn wounds. Artif. Organs 2008, 32 (12), 92531.

(7) Maharlooei, M. K.; Bagheri, M.; Solhjou, Z.; Jahromi, B. M.; Akrami, M.; Rohani, L.; Monabati, A.; Noorafshan, A.; Omrani, G. R. Adipose tissue derived mesenchymal stem cell (AD-MSC) promotes skin wound healing in diabetic rats. Diabetes Res. Clin. Pract. 2011, 93 (2), 228-34.

(8) Gimble, J. M.; Katz, A. J.; Bunnell, B. A. Adipose-derived stem cells for regenerative medicine. Circ. Res. 2007, 100 (9), 1249-60.

(9) Kim, W. S.; Park, B. S.; Sung, J. H.; Yang, J. M.; Park, S. B.; Kwak, S. J.; Park, J. S. Wound healing effect of adipose-derived stem cells: a critical role of secretory factors on human dermal fibroblasts. $J$. Dermatol. Sci. 2007, 48 (1), 15-24.

(10) Niemeyer, P.; Vohrer, J.; Schmal, H.; Kasten, P.; Fellenberg, J.; Suedkamp, N. P.; Mehlhorn, A. T. Survival of human mesenchymal stromal cells from bone marrow and adipose tissue after xenogenic transplantation in immunocompetent mice. Cytotherapy 2008, 10 (8), 784-95.

(11) Altman, A. M.; Yan, Y.; Matthias, N.; Bai, X.; Rios, C.; Mathur, A. B.; Song, Y. H.; Alt, E. U. IFATS collection: Human adiposederived stem cells seeded on a silk fibroin-chitosan scaffold enhance wound repair in a murine soft tissue injury model. Stem Cells 2009, 27 (1), 250-8.

(12) Wu, Y.; Chen, L.; Scott, P. G.; Tredget, E. E. Mesenchymal stem cells enhance wound healing through differentiation and angiogenesis. Stem Cells 2007, 25 (10), 2648-59.

(13) Aoki, S.; Toda, S.; Ando, T.; Sugihara, H. Bone marrow stromal cells, preadipocytes, and dermal fibroblasts promote epidermal regeneration in their distinctive fashions. Mol. Biol. Cell 2004, 15 (10), 4647-57.

(14) Lin, Y. C.; Grahovac, T.; Oh, S. J.; Ieraci, M.; Rubin, J. P.; Marra, K. G. Evaluation of a multi-layer adipose-derived stem cell sheet in a full-thickness wound healing model. Acta Biomater. 2012, 9 (2), 52435250.

(15) Fu, X.; Fang, L.; Li, X.; Cheng, B.; Sheng, Z. Enhanced woundhealing quality with bone marrow mesenchymal stem cells autografting after skin injury. Wound Repair Regen. 2006, 14 (3), 325-35.

(16) Kern, S.; Eichler, H.; Stoeve, J.; Kluter, H.; Bieback, K. Comparative analysis of mesenchymal stem cells from bone marrow, umbilical cord blood, or adipose tissue. Stem Cells 2006, 24 (5), 1294301.

(17) Suh, W.; Kim, K. L.; Kim, J. M.; Shin, I. S.; Lee, Y. S.; Lee, J. Y.; Jang, H. S.; Lee, J. S.; Byun, J.; Choi, J. H.; Jeon, E. S.; Kim, D. K. Transplantation of endothelial progenitor cells accelerates dermal wound healing with increased recruitment of monocytes/macrophages and neovascularization. Stem Cells 2005, 23 (10), 1571-8.

(18) Wu, Y.; Zhao, R. C.; Tredget, E. E. Concise review: bone marrow-derived stem/progenitor cells in cutaneous repair and regeneration. Stem Cells 2010, 28 (5), 905-15.

(19) Yamato, M.; Utsumi, M.; Kushida, A.; Konno, C.; Kikuchi, A.; Okano, T. Thermoresponsive culture dishes allow the intact harvest of multilayered keratinocyte sheets without Dispase by reducing temperature. Tissue Eng. 2001, 7 (4), 473-80.

(20) Masuda, S.; Shimizu, T.; Yamato, M.; Okano, T. Cell sheet engineering for heart tissue repair. Adv. Drug Delivery Rev. 2008, 60 (2), 277-85.

(21) Yang, J.; Yamato, M.; Shimizu, T.; Sekine, H.; Ohashi, K.; Kanzaki, M.; Ohki, T.; Nishida, K.; Okano, T. Reconstruction of functional tissues with cell sheet engineering. Biomaterials 2007, 28 (34), 5033-43.

(22) Okano, T.; Yamada, N.; Sakai, H.; Sakurai, Y. A novel recovery system for cultured cells using plasma-treated polystyrene dishes grafted with poly( $\mathrm{N}$-isopropylacrylamide). J. Biomed. Mater. Res. 1993, 27 (10), 1243-51.
(23) Sun, G.; Zhang, X.; Shen, Y. I.; Sebastian, R.; Dickinson, L. E.; Fox-Talbot, K.; Reinblatt, M.; Steenbergen, C.; Harmon, J. W.; Gerecht, S. Dextran hydrogel scaffolds enhance angiogenic responses and promote complete skin regeneration during burn wound healing. Proc. Natl. Acad. Sci. U.S.A. 2011, 108 (52), 20976-81.

(24) Wong, V. W.; Rustad, K. C.; Glotzbach, J. P.; Sorkin, M.; Inayathullah, M.; Major, M. R.; Longaker, M. T.; Rajadas, J.; Gurtner, G. C. Pullulan hydrogels improve mesenchymal stem cell delivery into high-oxidative-stress wounds. Macromol. Biosci. 2012, 11 (11), 145866.

(25) Wong, V. W.; Rustad, K. C.; Galvez, M. G.; Neofytou, E.; Glotzbach, J. P.; Januszyk, M.; Major, M. R.; Sorkin, M.; Longaker, M. T.; Rajadas, J.; Gurtner, G. C. Engineered pullulan-collagen composite dermal hydrogels improve early cutaneous wound healing. Tissue Eng. Part A 2010, 17 (5-6), 631-44.

(26) Chan, R. K.; Zamora, D. O.; Wrice, N. L.; Baer, D. G.; Renz, E. M.; Christy, R. J.; Natesan, S. Development of a vascularized skin construct using adipose-derived stem cells from debrided burned skin. Stem Cells Int. 2012, 2012, 841203.

(27) Lee, P. Y.; Cobain, E.; Huard, J.; Huang, L. Thermosensitive hydrogel PEG-PLGA-PEG enhances engraftment of muscle-derived stem cells and promotes healing in diabetic wound. Mol. Ther. 2007, 15 (6), 1189-94.

(28) Sekine, H.; Shimizu, T.; Dobashi, I.; Matsuura, K.; Hagiwara, N.; Takahashi, M.; Kobayashi, E.; Yamato, M.; Okano, T. Cardiac cell sheet transplantation improves damaged heart function via superior cell survival in comparison with dissociated cell injection. Tissue Eng., Part A 2011, 17 (23-24), 2973-80.

(29) Haraguchi, Y.; Shimizu, T.; Sasagawa, T.; Sekine, H.; Sakaguchi, K.; Kikuchi, T.; Sekine, W.; Sekiya, S.; Yamato, M.; Umezu, M.; Okano, T. Fabrication of functional three-dimensional tissues by stacking cell sheets in vitro. Nat. Protoc. 2012, 7 (5), 850-8.

(30) Obokata, H.; Yamato, M.; Tsuneda, S.; Okano, T. Reproducible subcutaneous transplantation of cell sheets into recipient mice. Nat. Protoc. 2011, 6 (7), 1053-9.

(31) Wicke, C.; Halliday, B.; Allen, D.; Roche, N. S.; Scheuenstuhl, H.; Spencer, M. M.; Roberts, A. B.; Hunt, T. K. Effects of steroids and retinoids on wound healing. Arch. Surg. 2000, 135 (11), 1265-70.

(32) Ananta, M.; Brown, R. A.; Mudera, V. A rapid fabricated living dermal equivalent for skin tissue engineering: an in vivo evaluation in an acute wound model. Tissue Eng., Part A 2012, 18 (3-4), 353-61.

(33) Liu, S.; Zhang, H.; Zhang, X.; Lu, W.; Huang, X.; Xie, H.; Zhou, J.; Wang, W.; Zhang, Y.; Liu, Y.; Deng, Z.; Jin, Y. Synergistic angiogenesis promoting effects of extracellular matrix scaffolds and adipose-derived stem cells during wound repair. Tissue Eng., Part A 2011, 17 (5-6), 725-39.

(34) Larouche, D.; Cuffley, K.; Paquet, C.; Germain, L. Tissueengineered skin preserving the potential of epithelial cells to differentiate into hair after grafting. Tissue Eng., Part A 2011, 17 (56), 819-30.

(35) Dominici, M.; Le Blanc, K.; Mueller, I.; Slaper-Cortenbach, I.; Marini, F.; Krause, D.; Deans, R.; Keating, A.; Prockop, D.; Horwitz, E. Minimal criteria for defining multipotent mesenchymal stromal cells. The International Society for Cellular Therapy position statement. Cytotherapy 2006, 8 (4), 315-7.

(36) Murad, S.; Grove, D.; Lindberg, K. A.; Reynolds, G.; Sivarajah, A.; Pinnell, S. R. Regulation of collagen synthesis by ascorbic acid. Proc. Natl. Acad. Sci. U.S.A. 1981, 78 (5), 2879-82.

(37) Vermette, M.; Trottier, V.; Menard, V.; Saint-Pierre, L.; Roy, A.; Fradette, J. Production of a new tissue-engineered adipose substitute from human adipose-derived stromal cells. Biomaterials 2007, 28 (18), 2850-60.

(38) Wong, V. W.; Sorkin, M.; Glotzbach, J. P.; Longaker, M. T.; Gurtner, G. C. Surgical approaches to create murine models of human wound healing. J. Biomed. Biotechnol. 2011, 2011, 969618.

(39) Stephens, F. O.; Dunphy, J. E.; Hunt, T. K. Effect of delayed administration of corticosteroids on wound contraction. Ann. Surg. 1971, 173 (2), 214-8. 
(40) Kim, W. S.; Park, B. S.; Sung, J. H. The wound-healing and antioxidant effects of adipose-derived stem cells. Expert Opin. Biol. Ther. 2009, 9 (7), 879-87.

(41) Prockop, D. J. "Stemness" does not explain the repair of many tissues by mesenchymal stem/multipotent stromal cells (MSCs). Clin. Pharmacol. Ther. 2007, 82 (3), 241-3.

(42) Natesan, S.; Wrice, N. L.; Baer, D. G.; Christy, R. J. Debrided skin as a source of autologous stem cells for wound repair. Stem Cells 2011, 29 (8), 1219-30.

(43) Ebrahimian, T. G.; Pouzoulet, F.; Squiban, C.; Buard, V.; Andre, M.; Cousin, B.; Gourmelon, P.; Benderitter, M.; Casteilla, L.; Tamarat, R. Cell therapy based on adipose tissue-derived stromal cells promotes physiological and pathological wound healing. Arterioscler. Thromb. Vasc. Biol. 2009, 29 (4), 503-10.

(44) Phinney, D. G. Biochemical heterogeneity of mesenchymal stem cell populations: clues to their therapeutic efficacy. Cell Cycle 2007, 6 (23), 2884-9.

(45) Rehman, J.; Traktuev, D.; Li, J.; Merfeld-Clauss, S.; TemmGrove, C. J.; Bovenkerk, J. E.; Pell, C. L.; Johnstone, B. H.; Considine, R. V.; March, K. L. Secretion of angiogenic and antiapoptotic factors by human adipose stromal cells. Circulation 2004, 109 (10), 1292-8.

(46) Lugo, L. M.; Lei, P.; Andreadis, S. T. Vascularization of the dermal support enhances wound re-epithelialization by in situ delivery of epidermal keratinocytes. Tissue Eng., Part A 2010, 17 (5-6), 66575.

(47) Yannas, I. V.; Lee, E.; Orgill, D. P.; Skrabut, E. M.; Murphy, G. F. Synthesis and characterization of a model extracellular matrix that induces partial regeneration of adult mammalian skin. Proc. Natl. Acad. Sci. U.S.A. 1989, 86 (3), 933-7.

(48) Wearing, H. J.; Sherratt, J. A. Keratinocyte growth factor signalling: a mathematical model of dermal-epidermal interaction in epidermal wound healing. Math. Biosci. 2000, 165 (1), 41-62.

(49) Guo, L.; Yu, Q. C.; Fuchs, E. Targeting expression of keratinocyte growth factor to keratinocytes elicits striking changes in epithelial differentiation in transgenic mice. EMBO J. 1993, 12 (3), 973-86.

(50) Werner, S.; Peters, K. G.; Longaker, M. T.; Fuller-Pace, F.; Banda, M. J.; Williams, L. T. Large induction of keratinocyte growth factor expression in the dermis during wound healing. Proc. Natl. Acad. Sci. U.S.A. 1992, 89 (15), 6896-900.

(51) Andreadis, S. T.; Hamoen, K. E.; Yarmush, M. L.; Morgan, J. R. Keratinocyte growth factor induces hyperproliferation and delays differentiation in a skin equivalent model system. FASEB J. 2001, 15 (6), 898-906.

(52) Ohashi, K.; Yokoyama, T.; Yamato, M.; Kuge, H.; Kanehiro, H.; Tsutsumi, M.; Amanuma, T.; Iwata, H.; Yang, J.; Okano, T.; Nakajima, $Y$. Engineering functional two- and three-dimensional liver systems in vivo using hepatic tissue sheets. Nat. Med. 2007, 13 (7), 880-5.

(53) Sekiya, N.; Matsumiya, G.; Miyagawa, S.; Saito, A.; Shimizu, T.; Okano, T.; Kawaguchi, N.; Matsuura, N.; Sawa, Y. Layered implantation of myoblast sheets attenuates adverse cardiac remodeling of the infarcted heart. J. Thorac. Cardiovasc. Surg. 2009, 138 (4), 98593.

(54) Iwata, T.; Yamato, M.; Tsuchioka, H.; Takagi, R.; Mukobata, S.; Washio, K.; Okano, T.; Ishikawa, I. Periodontal regeneration with multi-layered periodontal ligament-derived cell sheets in a canine model. Biomaterials 2009, 30 (14), 2716-23. 